\title{
Mass Measurement and Top-Down HPLC/MS Analysis of Intact Monoclonal Antibodies on a Hybrid Linear Quadrupole Ion Trap-Orbitrap Mass Spectrometer
}

\author{
Pavel V. Bondarenko, ${ }^{\mathrm{a}}$ Tonya P. Second, ${ }^{\mathrm{b}}$ Vlad Zabrouskov, ${ }^{\mathrm{b}}$ \\ Alexander A. Makarov, ${ }^{\mathrm{c}}$ and Zhongqi Zhang ${ }^{\mathrm{a}}$ \\ ${ }^{a}$ Amgen Incorporated, Thousand Oaks, California, USA \\ $\mathrm{b}$ Thermo Fisher, San Jose, California, USA \\ ${ }^{\mathrm{c}}$ Thermo Fisher, Bremen, Germany
}

\begin{abstract}
Mass and top-down analyses of 150-kDa monoclonal immunoglobulin gamma (IgG) antibodies were performed on an Orbitrap analyzer. Three different sample delivery methods were tested including (1) infusion of an off-line desalted IgG sample using nano-electrospray; (2) on-line desalting followed by a step elution with a high percentage of organic solvent; and (3) reversed-phase HPLC separation and on-line mass and top-down analyses of disulfide isoforms of an IgG2 antibody. The accuracy of mass measurements of intact antibody was within $\pm 2 \mathrm{Da}(15 \mathrm{ppm})$. The glycoforms of intact IgG antibodies separated by $162 \mathrm{Da}$ were baseline resolved. In-source fragmentation of the intact antibodies produced mainly 115 residue fragments including $\mathrm{N}$-terminal variable domains of heavy and light chains. The sequence coverage (the number of cleavages) was greatly increased after reduction of disulfide bonds and HPLC/MS/MS analysis of light and heavy chains using collisioninduced dissociation in the ion trap of the LTQ-Orbitrap. This is an attractive alternative to peptide mapping for characterization and monitoring of post-translational modifications attributed to minimal sample preparation, high speed of the mass/top-down analysis, and relatively minor method-induced sample modifications. (J Am Soc Mass Spectrom 2009, 20, 1415-1424) (c) 2009 Published by Elsevier Inc. on behalf of American Society for Mass Spectrometry
\end{abstract}

$\mathrm{B}$ ecause of their predictable properties, controlled function and long circulation lifetime, monoclonal human immunoglobulin gamma (IgG) antibodies have emerged as a popular therapeutic modality $[1,2]$. This has resulted in a strong need for a highthroughput methods for analysis of different antibody drug candidates. Mass spectrometry has become one of the most powerful techniques for the structural characterization of monoclonal antibodies (mAbs) [3]. Traditionally, structural characterization of mAbs has been performed by a "bottom-up" approach after first digesting them to peptides [4-6]. Unfortunately, enzymatic digestion is a laborious, time-consuming process and it often introduces artificial modifications, such as cyclization of N-terminal glutamine and deamidation [5, 7]. Alternatively, protein molecular mass analysis is relatively fast, does not require lengthy sample preparation, and induces fewer, if any, modifications [8] compared with the peptide mapping [5, 7]. Analysis of intact

Address reprint requests to Dr. Pavel V. Bondarenko, Amgen, Department of Pharmaceutics, One Amgen Center Drive, MS 8-1-C, Thousand Oaks, CA 91320. E-mail: pavel.bondarenko@amgen.com monoclonal IgG antibodies and their large domains has been reported for matrix-assisted laser desorption/ ionization (MALDI) and electrospray ionization (ESI) sources and almost all mass analyzers including MALDI-time of flight (TOF) [9-11], ESI quadrupole (Q) [4, 12-14], ion trap [15], orthogonal TOF [8, 11, 16, 17], and the LTQ-Orbitrap during direct infusion [18]. Although a mass change in a population of $\mathrm{mAbs}$ can be used to monitor post-translational modifications [8], it cannot reveal the site of modification. For that purpose, fragmentation of intact proteins, also known as "topdown" mass spectrometry, has been developed during the past decade [19-25] using mainly high-resolving power Fourier transform ion cyclotron resonance (FTICR) mass spectrometers. Combination of intact protein mass measurement and top-down fragmentation analysis provided useful information about sites of posttranslational modifications (PTMs) in several studies including oxidation of viral prolyl-4-hydroxylase [20] and deamidation of ribonuclease A [23], to name a few. Discovery of the Orbitrap analyzer [26] and its first commercialization as a hybrid linear quadrupole ion trap-Orbitrap mass spectrometer (LTQ Orbitrap, Thermo 
Scientific, Bremen, Germany) provided many laboratories with the opportunity to achieve high mass resolution without a superconducting magnet and its special and expensive maintenance. The LTQ-Orbitrap was used for top-down analysis of intact monoclonal IgG antibodies [27] and their light and heavy chains [18] during direct infusion as well as on-line with HPLC [3]. In addition, top-down (tandem mass spectroscopy, MS/MS) analysis of antibody chains was performed using a q-TOF mass spectrometer during direct infusion [28], and on-line with HPLC using in-source N-terminal fragmentation on an orthogonal-TOF instrument [29]. During production and formulation of a $\mathrm{mAb}$, it is important to separate and quantify all isoforms of therapeutic antibody with different PTMs to further identify the sites of the modifications and their impact on potency, stability, and other critical attributes of the therapeutic molecules. To avoid time-consuming and costly fractionation and fraction collection and subsequent peptide mapping of the separated isoforms, it is very attractive to separate the intact protein isoforms by liquid chromatography and then perform on-line mass and top-down analyses to determine the sites of modifications and their abundances in one short assay. In the previous work by Zhang and Shah the modifications inside the variable domains of antibodies were successfully characterized by a simple top-down method on the Orbitrap analyzer [27]. However, mass measurement of the intact antibodies was usually performed on a separate TOF analyzer, limiting the throughput of the analysis. Therefore, it was desirable to combine the mass measurement and the top-down fragmentation analysis of a $\mathrm{mAb}$ in one automated procedure. This report describes a step toward implementation of such analysis of human monoclonal antibodies and their large subunits (light and heavy chains after reduction of disulfide bonds) using the LTQ Orbitrap. We report mass measurements and top-down fragmentation analyses of intact and reduced IgG antibodies on the LTQOrbitrap connected to reversed-phase HPLC.

\section{Experimental}

\section{Materials}

The recombinant monoclonal IgG1 and IgG2 antibodies analyzed in this study were produced and purified at Amgen using standard manufacturing procedures. Water and acetonitrile (ACN) were obtained from VWR International (West Chester, PA, USA). Formic acid (FA) and trifluoroacetic acid (TFA) were from Pierce (Rockford, IL, USA). Guanidine $\mathrm{HCl}(\mathrm{GdnHCl})$ was obtained from Mallinckrodt Baker (Phillipsburg, NJ, USA).

\section{Reduction and Alkylation Procedure}

One IgG2 antibody used in this study was reduced and alkylated according to the procedure described in Reh- der et al. [30]. Briefly, the antibody was diluted to 2 $\mathrm{mg} / \mathrm{mL}$ using a buffer including 7.5 M GdnHCl, $0.1 \mathrm{M}$ Tris- $\mathrm{HCl}$ (Sigma, St. Louis, MO, USA), and $1 \mathrm{mM}$ ethylenediaminetetraacetic acid (EDTA, Sigma) at $\mathrm{pH}=$ 7.5 to a volume of $0.5 \mathrm{~mL}$. The sample was reduced with dithiothreitol (DTT, Sigma) and alkylated with iodoacetic acid (IAA, Sigma). The buffer $(0.5 \mathrm{~mL})$ of the reduced and alkylated protein was exchanged into $1 \mathrm{~mL}$ of 10 $\mathrm{mM}$ sodium acetate (J. T. Baker, Phillipsburg, NJ, USA) solution at $\mathrm{pH}=5.0$ to a final protein concentration of $1 \mathrm{mg} / \mathrm{mL}$. Buffer exchange was performed using a NAP-5 gel-filtration column packed with Sephadex G-25 medium (Amersham Pharmacia Biotech, Orsay, France) following the manufacturer's recommendations. Alternatively, instead of using buffer exchange, the reduced and alkylated sample was simply diluted 2 -fold in $20 \mathrm{mM}$ sodium acetate $(\mathrm{pH}=5.0)$ to lower the $\mathrm{pH}$ and dilute guanidine- $\mathrm{HCl}$ to prevent its crystallization in the autosampler at $4-8^{\circ} \mathrm{C}$.

\section{Nano-ESI Infusion, Step Elution, and RP HPLC}

Three different sample delivery systems were implemented as follows. First, nano-ESI infusion was performed with a solution containing $3.5 \mu \mathrm{g} / \mu \mathrm{L}$ of antibody in a ratio of 50:49:1 of acetonitrile:water:formic acid, respectively. Second, step elution from a $10 \times 2$ $\mathrm{mm}$ (length $\times$ diameter) reversed-phase guard column (Vydac C-4) was carried out using 95\% acetonitrile, $0.02 \% \mathrm{TFA}$, and $0.08 \%$ formic acid at $0.2 \mathrm{~mL} / \mathrm{min}$. Third, reversed-phase (RP) chromatography of intact and reduced antibodies was performed on an Agilent 1100 Capillary HPLC system with a Zorbax SB300 $300-\AA$ pore size, $3.5-\mu \mathrm{m}$ particle size, $50 \times 1-\mathrm{mm}$ column maintained at $75{ }^{\circ} \mathrm{C}$, and $50 \mu \mathrm{L} / \mathrm{min}$ flow rate. The mobile phase was water with $0.1 \%$ (vol/vol) TFA (solvent A) and 90\% (vol/vol) n-propanol (Burdick \& Jackson, Muskegon, MI, USA), 9.9\% (vol/vol) water, and $0.1 \%$ (vol/vol) TFA (solvent B). The elution gradient was from 21 to $27 \%$ B over 20 min for intact and from 21 to $27 \%$ over $40 \mathrm{~min}$ for reduced mAbs. Other details of the RP HPLC were described elsewhere for analysis of intact [31, 32] as well as reduced and alkylated [30] mAbs. The column eluate was analyzed by the on-line UV detector at $214 \mathrm{~nm}$ and then directed to mass spectrometric analysis.

\section{LTQ Orbitrap}

For the analysis of intact mAbs using nano-ESI infusion, a Thermo Scientific Nanospray I source was used equipped with an emitter with an external conductive coating and a 4- $\mu$ m-diameter tip (PicoTip, New Objective, Inc., Woburn, MA, USA). The instrument was operated with a spray voltage of $1.4 \mathrm{kV}$, a capillary voltage of $35 \mathrm{~V}$, a capillary temperature of $250^{\circ} \mathrm{C}$, a tube lens voltage of $220 \mathrm{~V}$, an Orbitrap target value of $10^{6}$, and an Orbitrap resolving power of 15,000 (at $\mathrm{m} / \mathrm{z}=$ 400). For on-line analysis after step elution and RP 
HPLC, the LTQ Orbitrap mass spectrometer was equipped with the regular Ion Max ESI/API source (Thermo Scientific). The source was operated using elevated voltages and temperature in the atmospherevacuum interface: a capillary voltage of $140 \mathrm{~V}$, a tube lens of $250 \mathrm{~V}$, a temperature of $350{ }^{\circ} \mathrm{C}$, a sheath gas flow of 50 units, and an auxiliary gas flow of 8 units. One scan included three microscans with maximum injection time of $500 \mathrm{~ms}$. Other Orbitrap parameters optimal for IgG mass measurement used a resolving power of 7500 (at $m / z=400$ ), an automatic gain control (AGC) target value of $10^{6}$, and each scan including five microscans with the Fourier transform mass spectrometry (FTMS) maximum injection time of $500 \mathrm{~ms}$. The method included one FTMS high mass range scan within $\mathrm{m} / \mathrm{z}$ 1000-3500 for mass measurement and one FTMS normal mass range scan within $m / z$ 400-2000 with source fragmentation energy of $75 \mathrm{~V}$ for top-down fragmentation analysis. The fragmentation spectra were acquired at the instrument-resolving power of 60,000 (at $\mathrm{m} / \mathrm{z}=$ 400), an AGC target value of $10^{6}$ and each scan including five microscans with the FTMS maximum injection time of $500 \mathrm{~ms}$. Data acquisition for the molecular ions and fragments was performed in profile mode. Although abundant fragments were observed during insource collision-induced dissociation (CID) of intact antibodies, better sequence coverage for light and heavy chains was usually achieved when a true MS/MS (isolation and fragmentation of parent ions in the ion trap) was performed because of reduced interference of an MS/MS experiment.

For analysis of heavy and light chains, the atmospherevacuum interface throughput of the Ion Max ESI/API source was optimal with the capillary at $14 \mathrm{~V}$, tube lens at $120 \mathrm{~V}$ and temperature at $350{ }^{\circ} \mathrm{C}$. In-trap CID was used because it produced better fragmentation and sequence coverage compared with in-source dissociation. The method included one Orbitrap (FTMS) high mass range scan $(\mathrm{m} / \mathrm{z}=600-3000)$ with resolving power of $60,000($ at $m / z=400)$ and an AGC target value of $5 \times$ $10^{5}$ followed by one MS/MS normal mass range scan $(m / z=350-2000)$ with the same Orbitrap resolving power and an AGC target value of $5 \times 10^{5}$. Each scan included five microscans with the FTMS maximum injection time of $1500 \mathrm{~ms}$. The MS/MS scan was performed using an isolation width of $m / z 100$ around the parent ions with $m / z=990$. The isolated multiply charged ions were activated using the default CID parameters of 30-ms activation time, a 35\% normalized collision energy, and an activation $q$ of 0.25 . All work was conducted in the positive-ion mode.

For nano-ESI infusion and step-elution experiments, the Orbitrap analyzer was calibrated in the high mass range (maximum $\mathrm{m} / \mathrm{z}$ value of 4000 ) using the calibrant PPG2700 and sodium-TFA cluster ions, respectively. For RP HPLC/MS studies, the Orbitrap analyzer was calibrated in the normal mass range (maximum $\mathrm{m} / \mathrm{z}$ value of 2000) using the Ultramark calibrant. This calibration was used for both the high mass range MS scans and normal mass range in-source and in-trap MS/MS fragmentation scans.

\section{Data Analysis Software}

MagTran [33] and ProMass software programs were used for deconvolution of ESI mass spectra. A program developed at Amgen, MassAnalyzer, was used to automatically assign the fragment ions [34, 35] and to prepare the fragment coverage map [27]. It was also used to calculate the theoretical average masses of the antibodies and their fragments, using atomic weights of elements from organic sources listed in reference [3].

\section{Results and Discussion}

Three different sample delivery approaches were evaluated for intact $\mathrm{mAb}$ mass measurement including direct infusion of an off-line desalted IgG sample using nano-electrospray (Figure 1); on-line desalting followed by a step elution with acetonitrile from a short guard column (Figure 2) and reversed-phase HPLC separation of disulfide isoforms of an IgG2 antibody (Figure 3 and Supplemental Figures S1 and S2, which can be found in the electronic version of this article) and also reduced antibody (Figures 4-6).

\section{IgG Mass Measurements Using Direct Infusion}

For direct infusion, the capillary voltage was set relatively low $(35 \mathrm{~V})$ to avoid significant fragmentation of the molecular ions. Only minor fragment ions appeared below $m / z=1700$ (Figure 1a). The higher tube lens voltage $(220 \mathrm{~V})$ helped transmitting the higher $\mathrm{m} / \mathrm{z}$ value ions. An Orbitrap resolving power of 15,000 gave the optimal resolution of the glycosylation forms. $\mathrm{Hu}-$ man IgG antibodies are glycoproteins containing two identical copies of light chain (LC) and heavy chain (HC) connected by interchain disulfide bonds (Supplemental Figure S2). The heavy chain has one conserved N-glycosylation site at asparagine residue 297 (N297) according to the Edelman numbering system. The glycosylation profile typically consists of two biantennary glycans with a variable number of terminal galactose residues [typically from zero (G0) to four (G4), Figure 1c]. The natural variability in terminal galactose sugar residues resulted in peaks that were $162 \mathrm{Da}$ apart (Figure $1 b$ and $c$ ). The oligosaccharides of IgG molecules do not contain (or contain only a minor percentage) of terminal sialic acid residues. A minor percentage of high mannose, defucosylated and other glycoforms of mAbs were previously reported $[30,36]$ and may contribute to the background peaks in Figure 1c.

\section{IgG Mass Measurements Using Step Elution}

Step elution from a short cartridge provided convenience of on-line desalting and automatic analysis of multiple samples (Figure 2). After directing salts to 
waste within the first $4 \mathrm{~min}$, the percentage of acetonitrile through the column was stepped from 0 to $95 \%$ and the eluting antibody molecules were ionized and
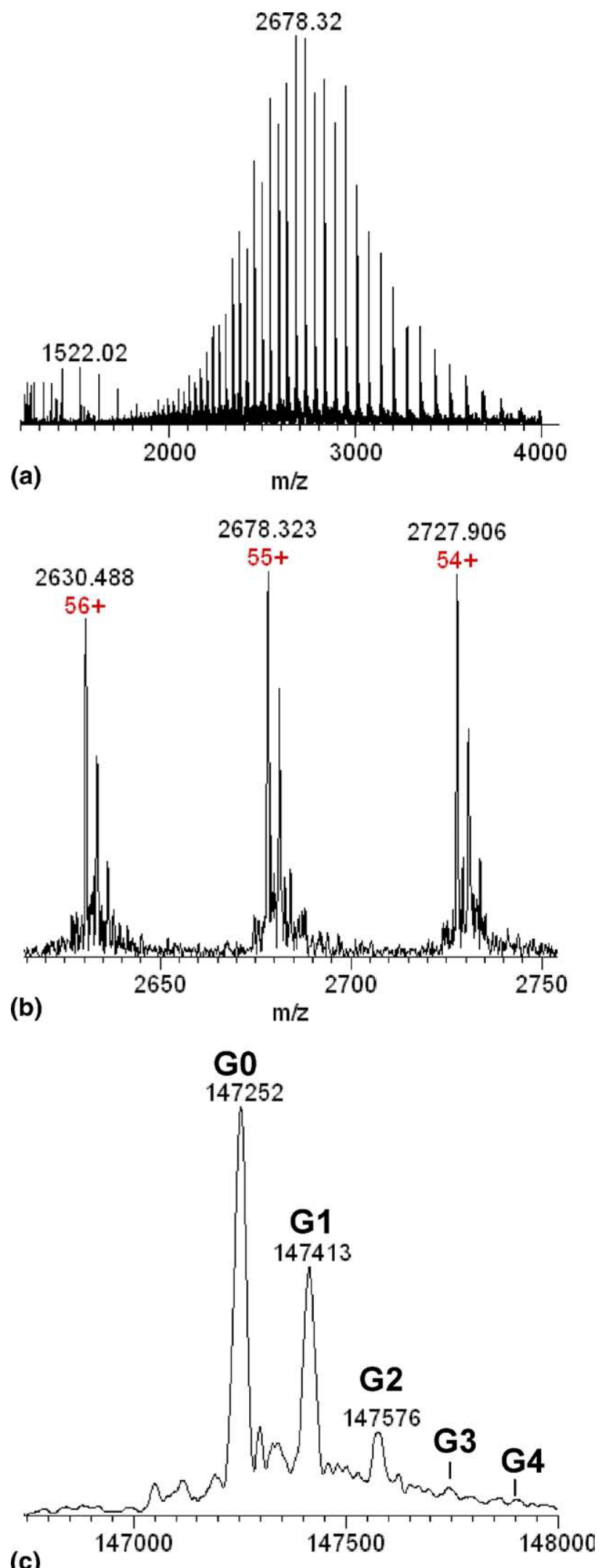

detected by the on-line Orbitrap. Sensitivity and mass spectrometric resolution during the step elution were better than that during the RP HPLC separation, with slow increase of organic solvent described in the following text, probably because of the higher percentage of organic solvent (95\% acetonitrile) and the lower percentage of TFA $(0.02 \%)$ during the step elution, which created more favorable electrospray conditions. IgG1 and IgG2 antibodies typically precipitate in solutions containing $>40 \%$ of organic solvent and $\mathrm{pH}>3$. Therefore a low $\mathrm{pH}(\mathrm{pH}<2.5)$ is required to maintain the antibodies in solution with a high percentage of organic solvent.

\section{Mass Measurement and Top-Down Fragmentation Analysis of IgG2 Disulfide Isoforms after RP HPLC Separation}

The utility of the RP HPLC/MS analysis was illustrated by measuring masses of disulfide isoforms of an IgG2 antibody (Figure 3). Deconvoluted ESI mass spectra of the reversed-phase peaks 1-4 of the intact monoclonal IgG2 antibody (Figure 3c) revealed identical mass values within the $\pm 2 \mathrm{Da}$ measurement error for the IgG2 disulfide isoforms, supporting previous findings [31, $37,38]$. Using nonreduced peptide mapping of collected fractions, the peaks were previously identified as having the same amino acid sequence and glycosylation, but different disulfide structure at the hinge [37, 38]. The measured masses were within 2-4 Da from the theoretical mass of $147,250 \mathrm{Da}$ using atomic weights of elements from organic sources listed in Zhang et al. [3].

As expected, formic acid instead of TFA in the mobile phases (used in the nano-ESI and step-elution sample delivery systems) increased the ion abundance and mass spectrometric resolution, but decreased the HPLC resolution of the IgG2 disulfide isoforms and the heavy chain $\mathrm{N}$-terminal forms (described in the following text). The step-elution option is not designed to separate the sample components chromatographically (only to desalt them), and thus it does not require a UV detector to quantify the components. Therefore, for better sensitivity, it can be implemented in micro- and nanoflow formats, for example by using the Advance Plug and Play Nanospray source (Michrom Bioresources, Inc., Auburn, CA, USA). The nano-ESI sample delivery option produced the highest intensity (per injected $\mu \mathrm{g}$ ) and resolution mass spectrum because of

Figure 1. Intact IgG using nano-ESI infusion; the average of 1000 scans for $5 \mathrm{~min}$ of static nano-electrospray; $0.9 \mu \mathrm{g}$ was infused. (a) ESI mass spectrum of an intact monoclonal IgG antibody with a theoretical molecular mass of $147,250 \mathrm{Da}$ for the G0 glycoform. The Orbitrap resolving power was 15,000 at $\mathrm{m} / \mathrm{z} 400$. (b) A section of ESI mass spectrum exhibiting multiply charged ions of intact antibody with 54, 55, and 56 protons. (c) Deconvoluted ESI mass spectrum of the IgG. Deconvolution was performed using ProMass software program. 

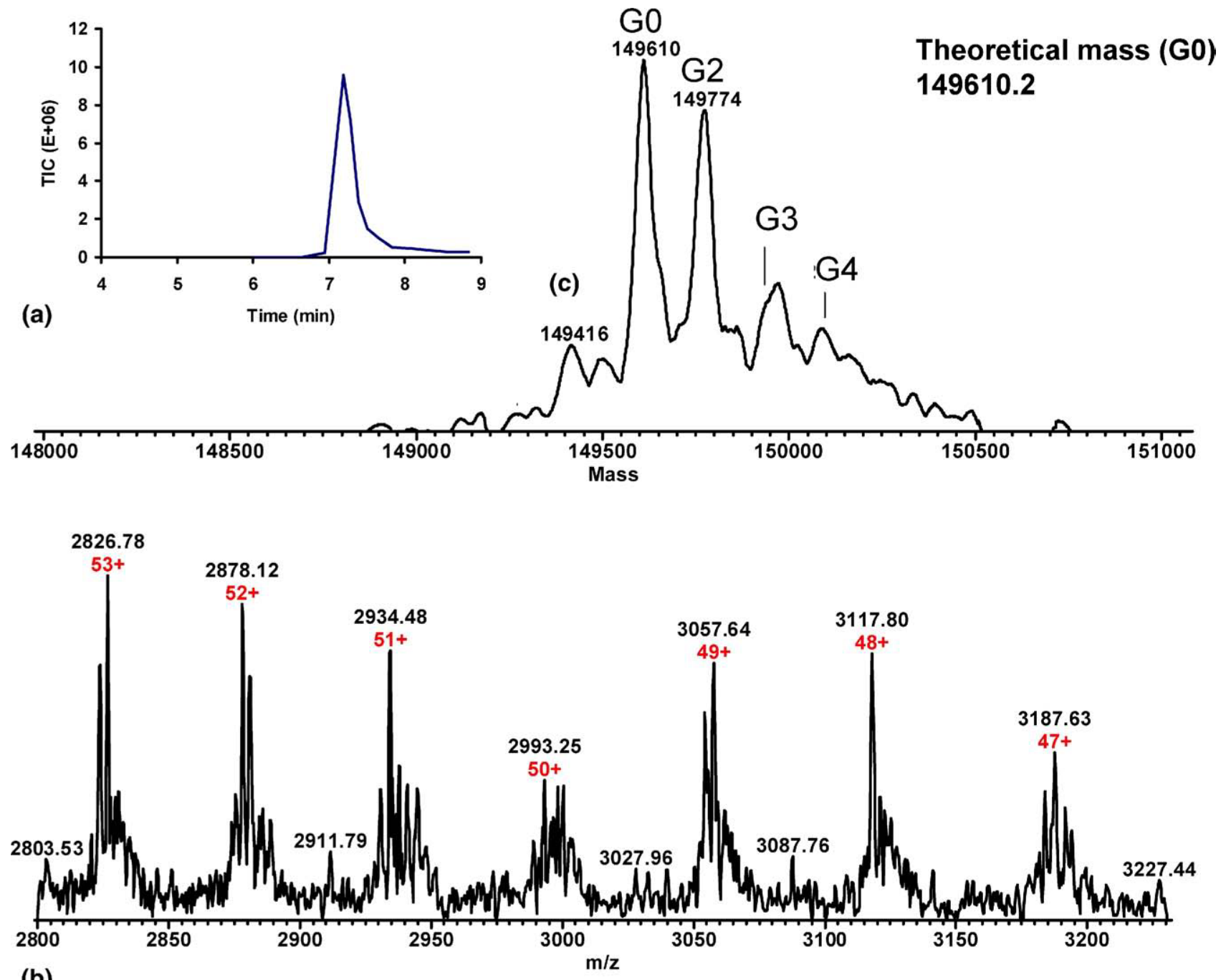

(b)

Figure 2. Intact $\mathrm{IgG}$ after step elution; $2 \mu \mathrm{g}$ was injected. (a) A total-ion chromatographic peak profile after a step elution from a $2 \times 10$-mm reversed-phase guard column. (b) A section of ESI mass spectrum used for deconvolution. (c) Deconvoluted ESI mass spectrum using MagTran software program. The theoretical molecular mass is 149,610.2 Da for the G0 glycoform.

the most effective droplet evaporation, ion formation, and declustering inherent to the nano-ESI process.

In addition to the accurate mass, the top-down fragmentation information was obtained by in-source CID within the same HPLC/MS run (Supplemental Figure S1). Although a detailed assessment of fragmentation differences for the disulfide isoforms is still under way and will be presented in a future publication, several general features of the top-down fragmentation analysis should be summarized here. The fragmentation pattern of this IgG2 was similar to the fragmentation pattern of another IgG2 antibody published previously [27] and several other IgG1 and IgG2 antibodies analyzed in the course of this study (data not shown). The most prominent fragments were large $12-\mathrm{kDa}$ fragment ions containing $\mathrm{N}$-terminal variable regions of the light and heavy chains (Supplemental Figure S1), similar to the fragments of the other $\mathrm{mAb}$ described in
Zhang and Shah [27]. The cleavage sites for these fragments were located in the loop between the variable and conserved domains of light and heavy chains shown in red in Supplemental Figure S2. Supplemental Figure S2 shows the most complete available highresolution $(2.7 \AA$, Protein Data Bank entry 1HZH) crystal structure of an IgG1 antibody [39]. Because no crystal structure of any IgG2 has been obtained to date, we used this available IgG1 crystal structure to visualize the cleavage sites. IgG2 and $\operatorname{IgG1}$ are very similar in primary structure and several other biochemical properties, strongly suggesting similarity in three-dimensional structure as well. Several IgG1 and IgG2 antibodies were analyzed in this study to show abundant fragmentation in the same conserved interdomain linker region shown in Supplemental Figure S1c.

It is interesting to note that fragmentation of the heavy chain was more abundant when acetonitrile was 


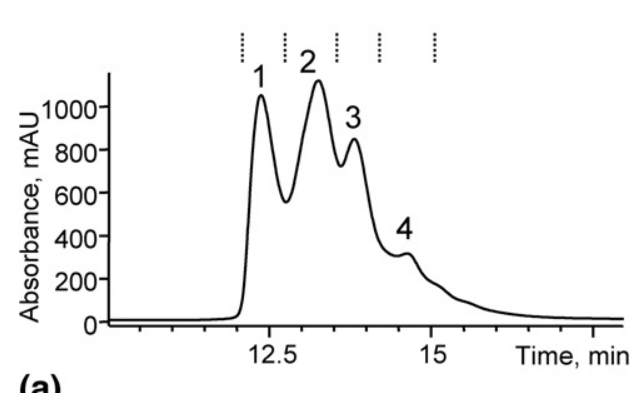

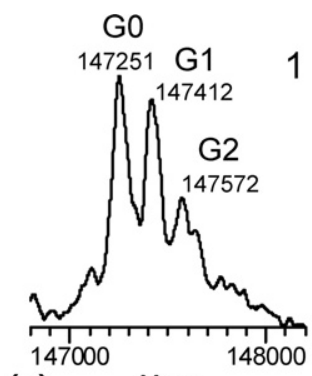

(c)

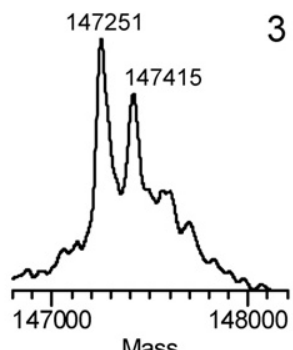

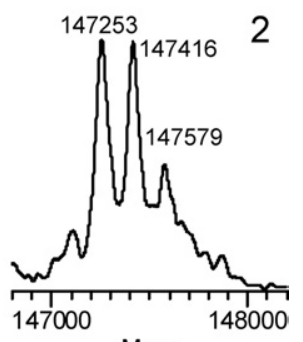

Mass

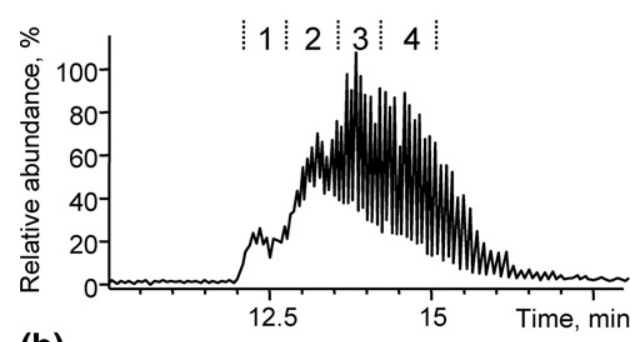

(b)

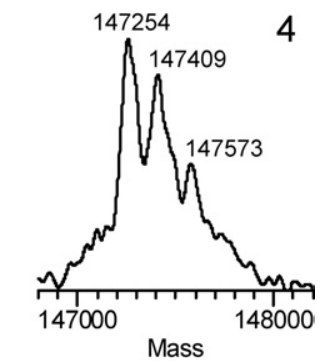

Figure 3. Mass measurement of IgG2 disulfide isoforms separated by RP HPLC; $4 \mu \mathrm{g}$ injected. (a) Reversed-phase chromatogram with UV absorbance at $214 \mathrm{~nm}$. (b) Total ion chromatogram featuring a series of MS scans (peaks) for mass measurement and in-source CID scans (valleys) for top-down analysis. (c) Deconvoluted ESI mass spectra of reversed-phase peaks 1-4 of intact monoclonal IgG2 antibody. Deconvolution was performed on MagTran.

used in the mobile phase (Supplemental Figure S1b) compared with the fragmentation during elution with $n$-propanol (Supplemental Figure S1a). The same column temperature and similar mobile-phase conditions were used in both cases. An even higher abundance of heavy chain fragments was obtained when an IgG2 antibody was step-eluted with $95 \%$ acetonitrile (data not shown). The observed difference in top-down
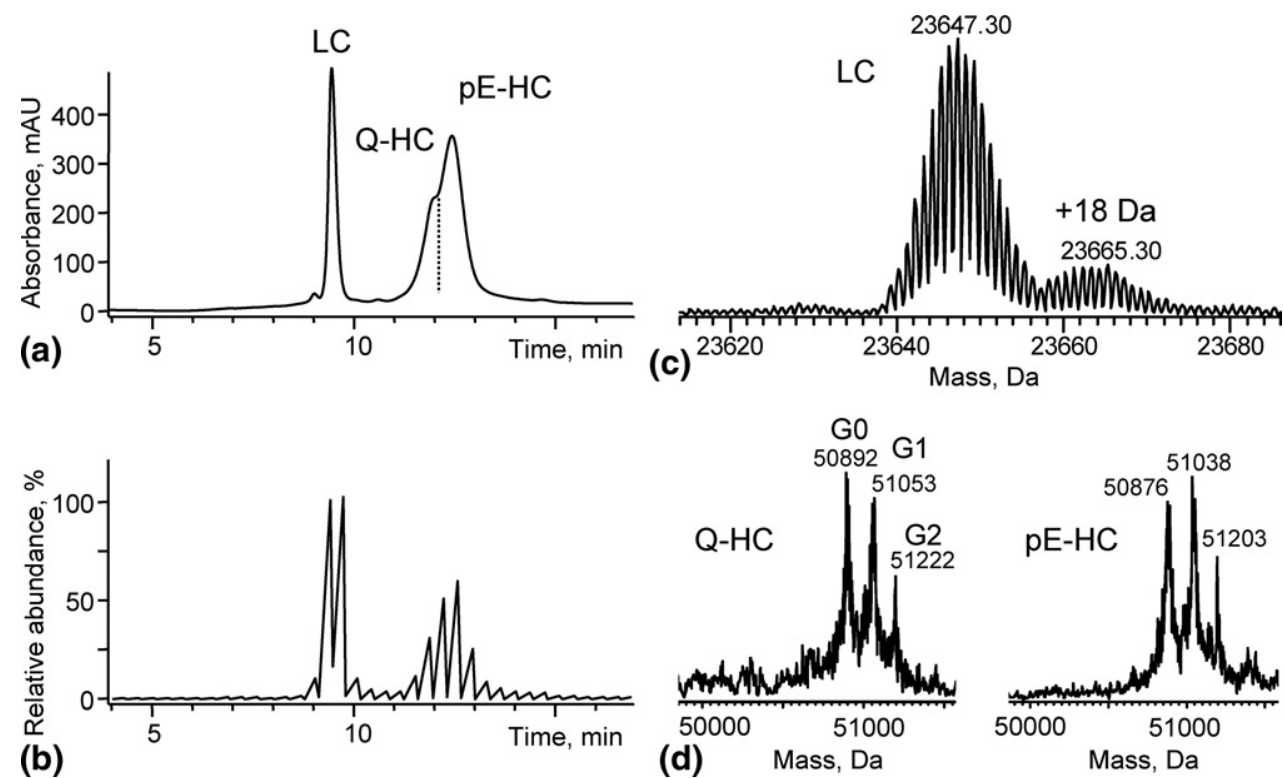

Figure 4. RP HPLC/MS/MS analysis of heavy and light chains after reduction and alkylation of an IgG2 antibody, $4 \mu \mathrm{g}$ was injected. (a) The UV chromatogram of separated light chain and the two partially separated variants of the heavy chain. (b) The total ion chromatogram containing a series of MS scans (peaks) for mass measurement and in-ion trap CID scans (valleys) for fragmentation analysis. The deconvoluted ESI mass spectra of (c) the light chain with theoretical molecular mass 23,647 Da and (d) the two heavy chain variants with theoretical mass values for Q-HC molecular mass of 50,893 Da and pE-HC of molecular mass of 50,876 Da for the G0 glycoform. Deconvolution was performed using MagTran software program. 


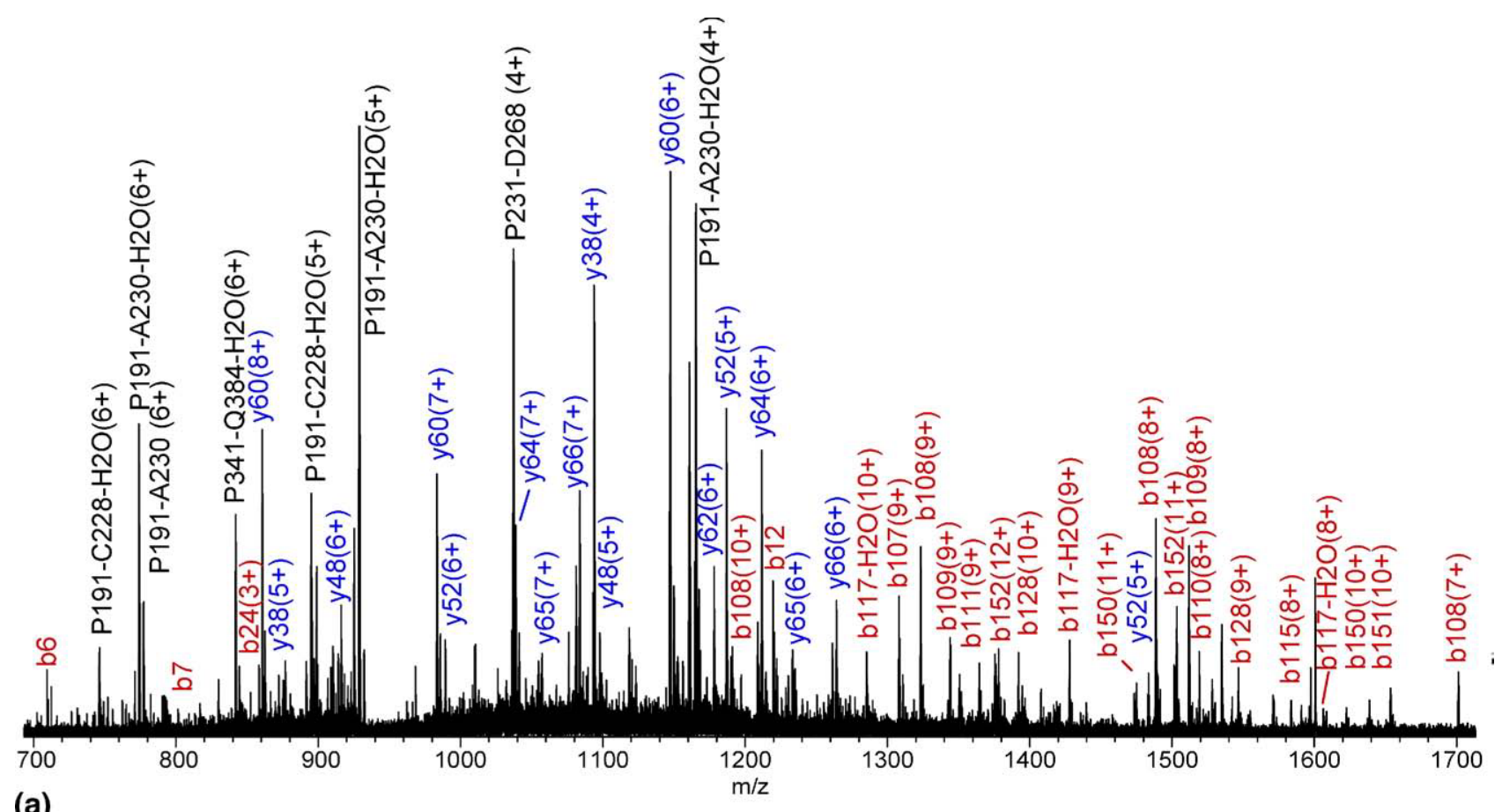

(a)

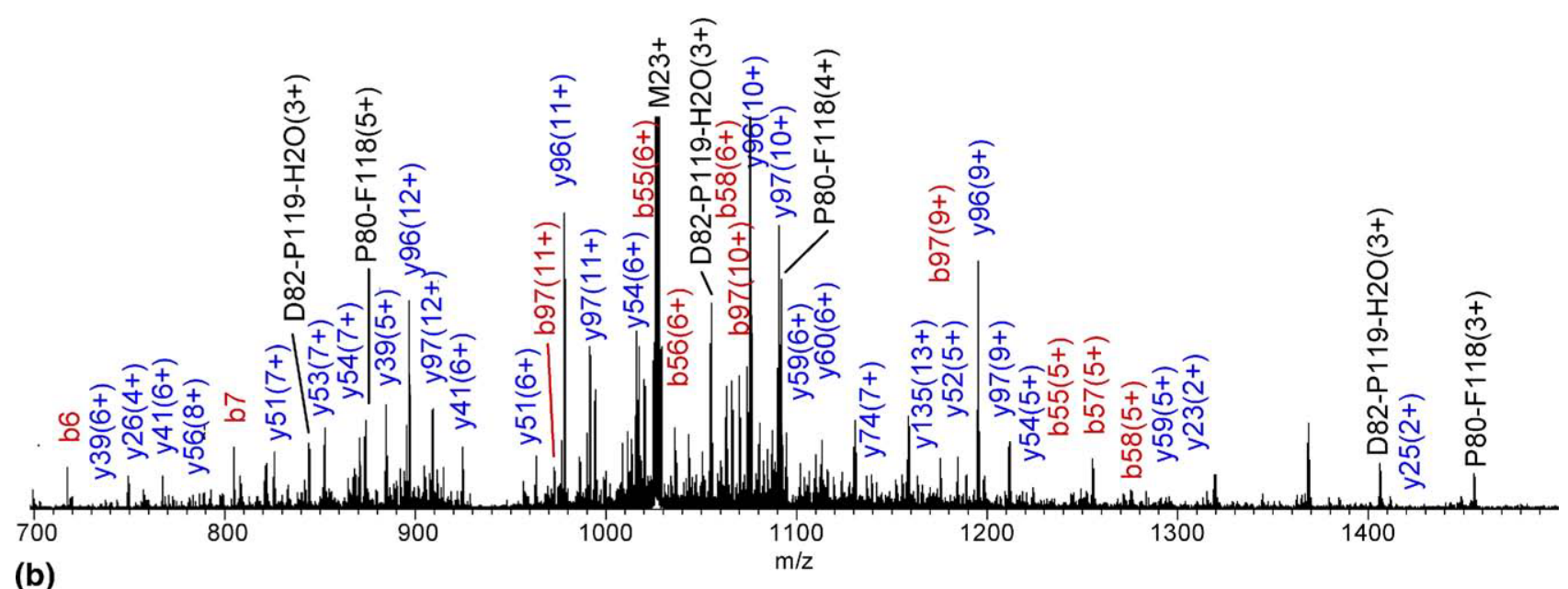

(b)

Figure 5. The fragmentation mass spectra of (a) the heavy chain variant $\mathrm{pE}-\mathrm{HC}$ and (b) the light chain. The CID of precursor ion mass $\mathrm{m} / \mathrm{z} 990$ with an isolation width $\mathrm{m} / \mathrm{z} 100 \mathrm{(m} / \mathrm{z} 940-1040$ as the isolation region) was performed in the ion trap during automated RP HPLC/MS/MS analysis of the reduced and alkylated IgG antibody. Ions labeled as a range indicate internal fragments. Only the most abundant fragment ions are labeled.

fragmentation pattern suggested that there may be a difference in folding of the molecules eluting from the column in the presence of acetonitrile and $n$ propanol. The higher abundance of the heavy chain fragments for acetonitrile suggests that eluted molecules were more loosely folded and are more susceptible to fragmentation in the case of acetonitrile. The ESI charge envelope was slightly shifted to lower $\mathrm{m} / \mathrm{z}$ and higher charge states for acetonitrile, supporting the hypothesis about the less folded and a more open structure in acetonitrile that carries a larger number of charges (protons).

\section{RP HPLC/MS/MS Analysis of Light and Heavy Chains after Reduction of Disulfide Bonds}

The interchain disulfide bonds between the heavy and light chains and also intrachain disulfide bonds inside the chains hold the structure together and suppress the fragmentation in IgG1 (data not shown) and IgG2 antibodies analyzed in this study. As a result, the top-down analysis of intact antibody molecules provided poor structural resolution with a relatively small number of cleavages. After reduction and alkylation of the disulfide bonds, HPLC/MS/MS analysis revealed 


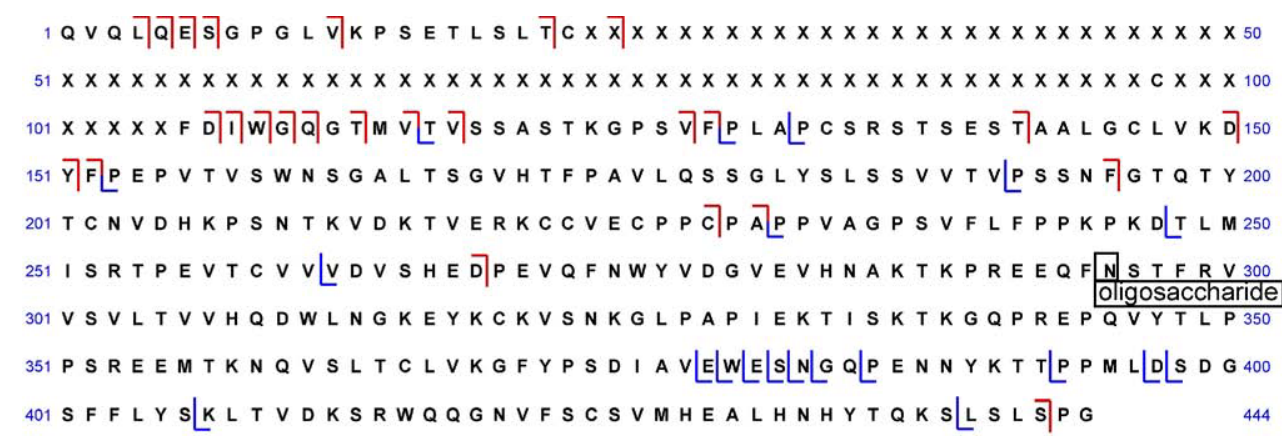

(a)

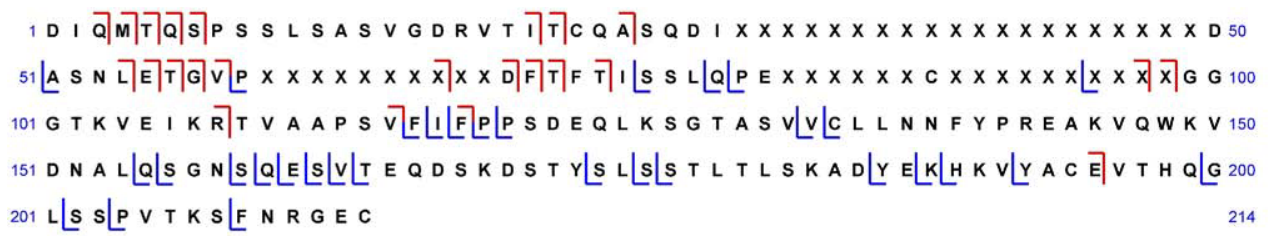

(b)

Figure 6. Amino acid sequence and fragment coverage maps of (a) the heavy chain and (b) the light chain of the reduced and alkylated IgG2 antibody generated by MassAnalyzer using the collisioninduced dissociation mass spectra in Figure $5 \mathrm{a}$ and $\mathrm{b}$. Only the sequences for the frames and conserved regions are provided. The letters " $\mathrm{X}$ " indicate complementarity determining regions unique only to this IgG2.

accurate masses of the light chain and two heavy chain variants and also increased the number of fragmentation sites and sequence coverage (Figures 4-6). Using an Orbitrap resolving power of 60,000 at $\mathrm{m} / \mathrm{z} 400$, isotopic resolution was achieved for the light chain with an average MW 23,647 (Figure 4c). Isotopes of the heavy chain could not be resolved at any resolving power during our studies. The mass spectra provided useful mass information and closely matched the calculated masses of heavy chain sequence with N-terminal glutamine (Q-HC, earlier eluting) and pyro-glutamic acid (pE-HC, later eluting in Figure 4). N-terminal cyclization is a common modification in IgGs and requires monitoring together with other modifications.

In-trap CID of the light and heavy chains (Figure 5) produced a better sequence coverage compared with that of in-source dissociation (not shown). The reason for the better sequence coverage when applying in-trap CID was likely a result of the reduced noise in the spectrum achieved by isolating an $\mathrm{m} / \mathrm{z} 100$ range of the precursor ions. The signal to noise was lower for the in-source CID, when all ions from the entire $\mathrm{m} / \mathrm{z}$ range were fragmented. Monoclonal antibodies against different antigens (targets) have different sequences and slightly different numbers of amino acid residues in the variable regions. Depending on the sequences of the variable regions, the masses of heavy and light chains are typically within $1 \mathrm{kDa}$ from the average mass values of $\sim 51$ and $\sim 23 \mathrm{kDa}$, respectively. The $\mathrm{m} / z 100$ isolation window from $\mathrm{m} / \mathrm{z}$ 940-1040 contained several abundant multiply charged ions of light chain (typically 23+, $24+, 25+$ ) and heavy chain (typically from $49+$ to $54+$ ). Selection of a large isolation width $(\mathrm{m} / \mathrm{z} 940-1040)$ was an attractive option because it provided a universal fragmentation parameter for light and heavy chains of different $m A b s$ (against different antigens) with different masses. This enabled an automated analysis of different IgG molecules without a prior knowledge of their masses. Using the maximal allowed isolation width of $m / z 100$, it was possible to reliably isolate and perform an automated MS/MS analysis of chains from any monoclonal $\mathrm{IgG}$ antibody. Using a large isolation width, however, calls for complete chromatographic separation of isoforms. For those experiments where complete chromatographic separation could not be achieved, an isoform of interest was isolated with a width of $\mathrm{m} / \mathrm{z} 10$ (data not shown), similar to the procedure described by Macek et al. [22]. This approach of using a narrow isolation window has an advantage, because it minimizes the need for chromatographic separation of protein variants. On the other hand, it requires a prior knowledge of the mass of protein isoform of interest, which is not practical for an automated high-throughput analysis of different antibodies.

The fragmentation spectrum of the heavy-chain variant with N-terminal glutamine (Q-HC, not shown here) was similar to the fragmentation mass spectrum of the heavy chain with $\mathrm{N}$-terminal pyro-glutamate ( $\mathrm{pE}-\mathrm{HC}$, Figure 5a). All b-fragment ions of Q-HC were $17 \mathrm{Da}$ heavier, pointing to the difference between the two heavy chain variants is on the $\mathrm{N}$-terminus. These findings unambiguously indicated that these two variants were attributed to the $\mathrm{N}$-terminal cyclization. In addition to the b-fragment ions containing $\mathrm{N}$-terminal glutamine, the fragmentation mass spectrum of the earlier eluting variant also contained abundant (b-17) Da frag- 
ment ions (not shown here). Two reasons may explain these peaks. They could be attributed to the incomplete separation of Q-HC and pE-HC or to the susceptibility of N-terminal glutamine to ammonia loss during fragmentation in the gas phase [30]. The chromatographic peaks for the heavy and light chains provided useful fragmentation coverage (Figure 6), which can be potentially used in other applications to identify sites of modifications, even if they are located in the middle of the chains. The majority of the fragments were represented by two or more charged forms (Figure 5). All fragment assignments shown here were automatically generated by MassAnalyzer software (using typically an 8 ppm mass accuracy threshold) and then validated manually by comparing their experimental isotope pattern to the calculated (by MassAnalyzer) theoretical isotope pattern.

\section{Conclusions}

Average masses of several 150-kDa intact monoclonal IgG antibody molecules were accurately determined using an Orbitrap analyzer within \pm 2 Da using the following delivery methods: (1) nano-ESI infusion, (2) step-elution, and (3) on-line reversed-phase HPLC. For online HPLC/MS analysis, both accurate mass and top-down fragmentation data were acquired within a short 20-min reversed-phase HPLC/MS run, using the Orbitrap analyzer alternating between a high mass range scan (for intact protein mass measurement) and a normal mass range scan with in-source fragmentation (for top-down analysis). The method can be used in pharmaceutical applications to establish identity and assess chemical modifications and mutations of therapeutic monoclonal IgG antibodies. The utility was illustrated by characterizing disulfide isoforms of an IgG2 antibody (Figure 3 and Supplemental Figures S1 and S2) and glutamine and pyro-glutamate variants of a heavy chain (Figures 4-6). The glycosylation profile, including several glycoforms with different numbers of terminal galactose residues $(n \times 162 \mathrm{Da})$, were determined from mass spectra of intact and reduced IgG antibodies. The use of acetonitrile instead of $n$-propanol in the mobile-phase increased abundance of heavy-chain top-down fragments, but decreased HPLC resolution.

An initial evaluation indicated that HPLC/MS/MS of light and heavy chains of a reduced and alkylated IgG increased the number of fragmentation sites and structural resolution of analysis (Figures 4-6), compared with the top-down analysis of intact IgG. This "middle-down" method was further developed and previously described [40]. Isotopic resolution of the light chain with an approximate MW $23 \mathrm{kDa}$ during on-line RP HPLC/MS analysis was achieved. Although isotopes for the heavy chain (MW $51 \mathrm{kDa}$ ) and intact antibody (MW $150 \mathrm{kDa}$ ) were unresolved at this time, we are looking forward to resolving them on new generations of Orbitrap analyzers. A future direction is aimed at obtaining more complete structural coverage (cleavage at every residue), which should be possible with additional dissociation methods and further development of Orbitrap technology.

\section{Acknowledgments}

The authors thank Jason Richardson for his contributions to the method development, and Joseph Phillips, David Brems, and Jim Thomas for fruitful discussions, support of this study, and valuable suggestions during review of the manuscript.

\section{Appendix A Supplementary Material}

Supplementary material associated with this article may be found in the online version at $10.1016 / \mathrm{j}$.jasms. 2009.03.020.

\section{References}

1. Reichert, J. M.; Rosensweig, C. J.; Faden, L. B.; Dewitz, M. C. Monoclonal Antibody Successes in the Clinic. Nat. Biotechnol. 2005, 23, 10731078 .

2. Maggon, K. Monoclonal Antibody “Gold Rush." Curr. Med. Chem. 2007, 14, 1978-1987.

3. Zhang, Z.; Chen, X.; Pan, H. Mass Spectrometry for Structural Characterization of Therapeutic Antibodies. Mass Spectrom. Rev. 2009, 28, 147-176.

4. Lewis, D. A.; Guzzetta, A. W.; Hancock, W. S.; Costello, M. Characterization of Humanized Anti-TAC, an Antibody Directed against the Interleukin 2 Receptor, Using Electrospray Ionization Mass Spectrometry by Direct Infusion, LC/MS, and MS/MS. Anal. Chem. 1994, 66, 585-595.

5. Bongers, J.; Cummings, J. J.; Ebert, M. B.; Federici, M. M.; Gledhill, L.; Gulati, D.; Hilliard, G. M.; Jones, B. H.; Lee, K. R.; Mozdzanowski, J.; Naimoli, M.; Burman, S. Validation of a Peptide Mapping Method for a Therapeutic Monoclonal Antibody: What Could We Possibly Learn about a Method We Have Run 100 Times? J. Pharm. Biomed. Anal. 2000, $21,1099-1128$.

6. Chelius, D.; Xiao, G.; Nichols, A. C.; Vizel, A.; He, B.; Dillon, T. M.; Rehder, D. S.; Pipes, G. D.; Kraft, E.; Oroska, A.; Treuheit, M. J. Bondarenko, P. V. Automated Tryptic Digestion Procedure for HPLC/ MS/MS Peptide Mapping of Immunoglobulin Gamma Antibodies in Pharmaceutics. J. Pharm. Biomed. Anal. 2008, 47, 285-294.

7. Chelius, D.; Rehder, D. S.; Bondarenko, P. V. Identification and Characterization of Deamidation Sites in the Conserved Regions of Human Immunoglobulin Gamma Antibodies. Anal. Chem. 2005, 77, 6004-6011.

8. Gadgil, H. S.; Pipes, G. D.; Dillon, T. M.; Treuheit, M. J.; Bondarenko, P. V. Improving Mass Accuracy of High Performance Liquid Chromatography/Electrospray Ionization Time-of-Flight Mass Spectrometry of Monoclonal Antibodies. J. Am. Soc. Mass Spectrom. 2006, 17, 867-872.

9. Alexander, A. J.; Hughes, D. E. Monitoring of IgG Antibody Thermal Stability by Micellar Electrokinetic Capillary Chromatography and Matrix-Assisted Laser Desorption/Ionization Mass Spectrometry. Anal. Chem. 1995, 67, 3626-3632.

10. Downard, K. M. Contributions of Mass Spectrometry to Structural Immunology. J. Mass Spectrom. 2000, 35, 493-503.

11. Beck, A.; Bussat, M. C.; Zorn, N.; Robillard, V.; Klinguer-Hamour, C.; Chenu, S.; Goetsch, L.; Corvaia, N.; van Dorsselaer, A.; Haeuw, J. F. Characterization by Liquid Chromatography Combined with Mass Spectrometry of Monoclonal Anti-IGF-1 Receptor Antibodies Produced in CHO and NS0 Cells. J. Chromatogr. B 2005, 819, 203-218.

12. Roberts, G. D.; Johnson, W. P.; Burman, S.; Anumula, K. R.; Carr, S. A. An Integrated Strategy for Structural Characterization of the Protein and Carbohydrate Components of Monoclonal Antibodies: Application to Anti-Respiratory Syncytial Virus MAb. Anal. Chem. 1995, 67, 36133625 .

13. Bennett, K. L.; Smith, S. V.; Lambrecht, R. M.; Truscott, R. J.; Sheil, M. M. Rapid Characterization of Chemically-Modified Proteins by Electrospray Mass Spectrometry. Bioconjug. Chem. 1996, 7, 16-22.

14. Masuda, K.; Yamaguchi, Y.; Kato, K.; Takahashi, N.; Shimada, I.; Arata, Y. Pairing of Oligosaccharides in the Fc Region of Immunoglobulin G. FEBS Lett. 2000, 473, 349-357.

15. Le, J. C.; Bondarenko, P. V. Trap for MAbs: Characterization of Intact Monoclonal Antibodies Using Reversed-Phase HPLC On-line with Ion-Trap Mass Spectrometry. J. Am. Soc. Mass Spectrom. 2005, 16, 307-311.

16. Verentchikov, A. N.; Ens, W.; Standing, K. G. Reflecting Time-of-Flight Mass Spectrometer with an Electrospray Ion Source and Orthogonal Extraction. Anal. Chem. 1994, 66, 126-133. 
17. Tito, M. A.; Miller, J.; Walker, N.; Griffin, K. F.; Williamson, E. D.; Despeyroux-Hill, D.; Titball, R. W.; Robinson, C. V. Probing Molecular Interactions in Intact Antibody: Antigen Complexes, an Electrospray Time-of-Flight Mass Spectrometry Approach. Biophys. J. 2001, 81, 35033509.

18. Zhang, J.; Katta, V. Top-Down Characterization of Therapeutic Antibody by High Resolution LTQ Orbitrap Mass Spectrometry. Proceedings of the 55th ASMS Conference on Mass Spectrometry and Allied Topics, Indianapolis, IN, June 3-7, 2007.

19. Kelleher, N. L.; Lin, H. Y.; Valaskovic, G. A.; Aaserud, D. I.; Fridriksson, E. K.; McLafferty, F. W. Top Down versus Bottom Up Protein Characterization by Tandem High-Resolution Mass Spectrometry. J. Am. Chem. Soc. 1999, 121, 806-812.

20. Ge, Y.; Lawhorn, B. G.; ElNaggar, M.; Sze, S. K.; Begley, T. P.; McLafferty, F. W. Detection of Four Oxidation Sites in Viral Prolyl-4hydroxylase by Top-Down Mass Spectrometry. Protein Sci. 2003, 12, 2320-2326.

21. Kruppa, G. H.; Schoeniger, J.; Young, M. M. A Top Down Approach to Protein Structural Studies Using Chemical Cross-Linking and Fourier Transform Mass Spectrometry. Rapid Commun. Mass Spectrom. 2003, 17, 155-162.

22. Macek, B.; Waanders, L. F.; Olsen, J. V.; Mann, M. Top-Down Protein Sequencing and MS3 on a Hybrid Linear Quadrupole Ion Trap-Orbitrap Mass Spectrometer. Mol. Cell. Proteomics 2006, 5, 949-958.

23. Zabrouskov, V.; Han, X.; Welker, E.; Zhai, H.; Lin, C.; van Wijk, K. J.; Scheraga, H. A.; McLafferty, F. W. Stepwise Deamidation of Ribonuclease A at Five Sites Determined by Top Down Mass Spectrometry. Biochemistry 2006, 45, 987-992.

24. Bogdanov, B.; Smith, R. D. Proteomics by FTICR Mass Spectrometry: Top Down and Bottom Up. Mass Spectrom. Rev. 2005, 24, 168-200.

25. Narayan, M.; Welker, E.; Zhai, H.; Han, X.; Xu, G.; McLafferty, F. W.; Scheraga, H. A. Detecting Native Folds in Mixtures of Proteins That Contain Disulfide Bonds. Nat. Biotechnol. 2008, 26, 427-429.

26. Makarov, A. A. Electrostatic Axially Harmonic Orbital Trapping: A High-Performance Technique of Mass Analysis. Anal. Chem. 2000, 72, $1156-1162$.

27. Zhang, Z.; Shah, B. Characterization of Variable Regions of Monoclonal Antibodies by Top-Down Mass Spectrometry. Anal. Chem. 2007, 79, 5723-5729.

28. Toler, K. N.; Johnson, K. A.; Rouse, J. C. Characterization of the Fragmentation Behavior of Light and Heavy Chain Ions from Recombinant Monoclonal Antibodies. Proceedings of the 53rd ASMS Conference on Mass Spectrometry and Allied Topics, San Antonio, TX, June 5-9, 2005.
29. Ren, D.; Pipes, G. D.; Hambly, D.; Bondarenko, P. V.; Treuheit, M. J.; Gadgil, H. S. Top-Down N-Terminal Sequencing of Immunoglobulin Subunits with Electrospray Ionization Time of Flight Mass Spectrometry. Anal. Biochem. 2008, 384, 42-48.

30. Rehder, D. S.; Dillon, T. M.; Pipes, G. D.; Bondarenko, P. V. ReversedPhase LC/MS Analysis of Reduced Monoclonal Antibodies in Pharmaceutics. J. Chromatogr. A 2006, 1102, 164-175.

31. Dillon, T. M.; Bondarenko, P. V.; Rehder, D. S.; Pipes, G. D.; Kleemann, G. R.; Ricci, M. S. Optimization of a Reversed-Phase LC/MS Method for Characterizing Recombinant Antibody Heterogeneity and Stability. J. Chromatogr. A 2006, 1120, 112-120.

32. Chu, G. C.; Chelius, D.; Xiao, G.; Khor, H. K.; Coulibaly, S.; Bondarenko, P. V. Accumulation of Succinimide in a Recombinant Monoclonal Antibody in Mildly Acidic Buffers under Elevated Temperatures. J. Pharm. Res. 2007, 24, 1145-1156.

33. Zhang, Z.; Marshall, A. G. A Universal Algorithm for Fast and Automated Charge State Deconvolution of Electrospray Mass-to-Charge Ratio Spectra. J. Am. Soc. Mass Spectrom. 1998, 9, 225-233.

34. Zhang, Z. Prediction of Low-Energy Collision-Induced Dissociation Spectra of Peptides. Anal. Chem. 2004, 76, 3908-3922.

35. Zhang, Z. De Novo Peptide Sequencing Based on a Divide-andConquer Algorithm and Peptide Tandem Spectrum Simulation. Anal. Chem. 2004, 76, 6374-6383.

36. Chen, X.; Flynn, G. C. Analysis of N-Glycans from Recombinant Immunoglobulin $\mathrm{G}$ by On-line Rreversed-Phase High-Herformance Liquid Chromatography/Mass Spectrometry. Anal. Biochem. 2007, 370, 147-161.

37. Wypych, J.; Li, M.; Guo, A.; Zhang, Z.; Martinez, T.; Allen, M.; Fodor, S. Kelner, D.; Flynn, G. C.; Liu, Y. D.; Bondarenko, P. V.; Speed-Ricci, M.; Dillon, T. M.; Balland, A. Human IgG2 Antibodies Display Disulfide Mediated Structural Isoforms. J. Biol. Chem. 2008, 283, 16194-16205.

38. Dillon, T. M.; Speed-Ricci, M.; Vezina, C.; Flynn, G. C.; Liu, Y. D. Rehder, D. S.; Plant, M.; Henkle, B.; Li, Y.; Varnum, B.; Wypych, J.; Balland, A.; Bondarenko, P. V. Structural and Functional Characterization of Disulfide Isoforms of the Human IgG2 Subclass. J. Biol. Chem. 2008, 283, 16206-16215.

39. Saphire, E. O.; Parren, P. W.; Pantophlet, R.; Zwick, M. B.; Morris, G. M.; Rudd, P. M.; Dwek, R. A.; Stanfield, R. L.; Burton, D. R.; Wilson, I. A. Crystal Structure of a Neutralizing Human IGG against HIV-1: A Template for Vaccine Design. Science 2001, 293, 1155-1159.

40. Richardson, J.; Hong, R.; Eris, T.; Zhang, Z. Middle-Down LC/MS Characterizations of Monoclonal IgG Antibodies. Proceedings of the 56th ASMS Conference on Mass Spectrometry and Allied Topics, Denver, CO, June 1-5, 2008. 